\section{Die kinetische Energie der Spaltungsfragmente}

Von W. Brunner und H. Paul

Kernphysikalisches Institut der Deutschen Akademie der Wissenschaften zu Berlin, Zeuthen

(Z. Naturforschg. 15 a, 1109-1110 [1960] ; eingeg. am 27. Oktober 1960)

In einer vorangehenden Arbeit ${ }^{1}$ wurde der Versuch unternommen, die experimentell bekannte Massenverteilung bei der Spaltung von $\mathrm{U}^{235}$ durch thermische Neutronen auf der Grundlage der Vorstellung einer schalenstrukturabhängigen Deformation der Fragmente „im Augenblick“ ihrer Entstehung zu erklären. Im folgenden soll gezeigt werden, daß sich aus demselben physikalischen Bild heraus auch der Verlauf der mittleren kinetischen Energie $T$ der Bruchstücke und im besonderen der darin zum Ausdruck kommende „magische Effekt" (Maxima von $T$ beim Auftreten magischer Spaltprodukte) verstehen läßt.

Die kinetische Energie $T$ ist offenbar gleich der Höhe des virtuellen Niveaus $\varepsilon$ des $Z$ weiteilchen-Systems $(\varepsilon$ enthält nicht die Anregungsenergie der Bruchstücke!), in der die Durchdringung der aus Cоulomв-Potential und Kernkraftpotential sich zusammensetzenden Potentialschwelle ${ }^{1}$

$$
V=\frac{Z_{1} Z_{2} e^{2}}{s}-V_{\mathrm{K}}^{0}\left(\alpha_{1}, \alpha_{2}\right) e^{-\mu(s-R)} \quad\left(s \geq s_{0}\right),
$$

- und damit der Zerfall des Zweiteilchensystems stattfindet. (Wir verwenden die gleichen Bezeichnungen wie früher ${ }^{1} ; s_{0}$ ist der Wert des Schwerpunktsabstandes $s$ der beiden Bruchstücke, bei dem letztere sich gerade berühren.)

$\varepsilon$ ist durch die Forderung definiert, daß die zugehörige Wellenfunktion $\psi(s)$ des Zweiteilchensystems für $s \rightarrow \infty$ eine reine auslaufende Welle darstellt. Wir halten die Annahme für plausibel, daß die Randbedingung für $\psi$ an der Stelle $s=s_{0}$, - die für die Berechnung von $\varepsilon$ notwendig ist und die wir explizit nicht kennen (sie wäre nur durch Lösung des A-Teilchen-Problems zu beschaffen) - , unabhängig vom Massenverhältnis $m$ ist, da die physikalische Situation (Kontakt der beiden Fragmente) unabhängig von $m$ jeweils die gleiche ist. Mit dieser Annahme ist dann $T$ in Abhängigkeit von $m$ bis auf eine $m$-unabhängige additive Konstante zu berechnen, wie sogleich näher ausgeführt werden soll.

Mit der Bezeichnung $s^{\prime} \equiv s-s_{0}$ läßt sich statt (1) schreiben

$$
V=\frac{Z_{1} Z_{2} e^{2}}{s_{0}+s^{\prime}}-\hat{V}_{\mathrm{K}} e^{-\mu s^{\prime}},
$$

wobei $-\hat{V}_{\mathrm{K}}$ das - in guter Näherung $m$-unabhängige Kernkraftpotential zweier sich berührender Fragmente bezeichnen soll. Der Wert von $\varepsilon$ wird hauptsächlich durch den Verlauf von $V$ für kleine Werte $s^{\prime}$ bestimmt, daher kann man in (2) den ersten Term nach Potenzen von $s^{\prime}$ entwickeln und die Entwicklung mit dem zweiten

1 W. Brunner u. H. PAUl, Z. Naturforschg. 15 a, 1018 [1960].
Glied abbrechen. Man erhält so

$$
V \simeq \frac{Z_{1} Z_{2} e^{2}}{s_{0}}-\frac{Z_{1} Z_{2} e^{2} s^{\prime}}{s_{0}{ }^{2}}-\hat{V}_{\mathrm{K}} e^{-\mu s^{\prime}} .
$$

Da die Variation von $Z_{1} Z_{2} / s_{0}{ }^{2}$ mit $m$ ebenfalls vernachlässigbar ist, unterscheidet sich der Potentialverlauf für zwei verschiedene Werte $m_{2}$ und $m_{1}$ nur durch die additive Konstante

$$
\begin{array}{r}
Z_{1}\left(m_{2}\right) Z_{2}\left(m_{2}\right) e^{2} \cdot s_{0}{ }^{-1}\left(m_{2}\right) \\
-Z_{1}\left(m_{1}\right) Z_{2}\left(m_{1}\right) e^{2} \cdot s_{0}{ }^{-1}\left(m_{1}\right) ;
\end{array}
$$

dasselbe gilt dann wegen der oben gemachten Annahme über die $m$-Unabhängigkeit der Randbedingung auch für die virtuellen Niveaus, das heißt

$$
\varepsilon\left(m_{2}\right)-\varepsilon\left(m_{1}\right)=\frac{Z_{1}\left(m_{2}\right) Z_{2}\left(m_{2}\right) e^{2}}{s_{0}\left(m_{2}\right)}-\frac{Z_{1}\left(m_{1}\right) Z_{2}\left(m_{1}\right) e^{2}}{s_{0}\left(m_{1}\right)}
$$

oder, unter Beachtung von

$$
s_{0}=\left(1+\alpha_{1}\right) R_{1}{ }^{0}+\left(1+\alpha_{2}\right) R_{2}{ }^{0}
$$

[entsprechend Gl. (4) von Anm. ${ }^{1}$,

$$
\varepsilon=\frac{Z_{1} Z_{2} e^{2}}{\left(1+a_{1}\right) R_{1}{ }^{0}+\left(1+a_{2}\right) R_{2}{ }^{0}}-\lambda,
$$

wobei $\lambda$ eine $m$-unabhängige Konstante ist.

Man erkennt aus der letzten Gleichung deutlich die Auswirkung des „magischen“ Deformationseffektes auf den Verlauf von $\varepsilon$ und damit auch von $T$ : Für ein Massenverhältnis $m$, bei dem eines der Bruchstücke magisch (bezüglich, seiner Neutronenzahl) ist, hat der Nenner in Gl. (4) wegen der geringen Deformation des magischen Fragments einen besonders kleinen Wert, daher zeigt die $T$-Kurve an dieser Stelle $m$ ein Maximum.

Die aus Gl. (4) (mit den Werten der Deformationsparameter $\alpha_{1}$ und $\alpha_{2}$ aus Anm. ${ }^{1}$, die sich aus der experimentellen Massenverteilung ergeben) für die Spaltung von $\mathrm{U}^{235}$ durch thermische Neutronen folgenden $T$-Werte sind in Abb. 1 aufgetragen. (Hierbei wurde $R_{1}{ }^{0}+R_{2}{ }^{0}=11,9 \cdot 10^{-13} \mathrm{~cm}$ und $\lambda=35,3 \mathrm{MeV}$ gesetzt.)

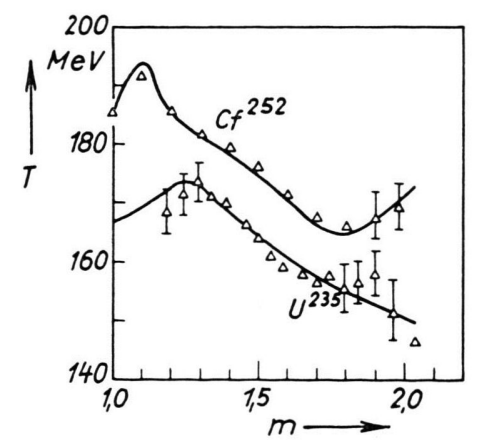

Abb. 1. Mittlere kinetische Energie $T$ der beiden Fragmente für die Spaltung von $U^{235}$ durch thermische Neutronen und für die spontane Spaltung von $\mathrm{Cf}^{252}$ als Funktion des Massenverhältnisses $m$. Kurven: theoretische Werte nach Gl. (4), $\triangle$ experimentelle Werte. 
Sie stimmen, wie aus der Abb. ersichtlich ist, gut mit den Meßwerten nach STEIN ${ }^{2}$ überein.

Als weiteres Beispiel soll die spontane Spaltung von $\mathrm{Cf}^{252}$ diskutiert werden. Zunächst berechnen wir (nach der Methode von Anm. ${ }^{1}$ ) aus der Spaltungswahrscheinlichkeit den Wert des Kernkraftpotentials $V_{\mathrm{K}}$ für ein festes $s$ in Abhängigkeit von $m$ (Abb. 2) und daraus

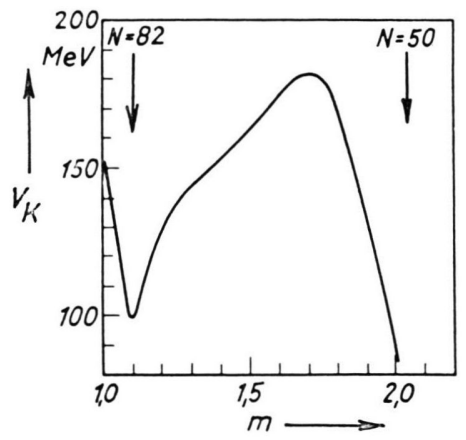

Abb. 2. Kernkraftwechselwirkung $V_{\mathrm{K}}$ für $s=14,0 \cdot 10^{-13} \mathrm{~cm}$ bei der spontanen Spaltung von $\mathrm{Cf}^{252}$ als Funktion des Massenverhältnisses (nach Anm. ${ }^{1}$ aus der Massenverteilung berechnet).

(ebenfalls nach Anm. ${ }^{1}$ ) die Deformation eines Bruchstückes in Abhängigkeit von seiner Neutronenzahl (Abb. 3). In beiden Kurven sind die „magischen Effekte" (Minima für den Fall, daß eines der Bruchstücke bezüglich seiner Neutronenzahl magisch ist $[N=50$ oder $N=82]$ ) deutlich erkennbar, wodurch die Überlegungen von Anm. ${ }^{1}$ eine weitere Stütze erfahren. Der aus der $\alpha^{2}$-Kurve der Abb. 3 nach Gl. (4) (mit $R_{1}{ }^{0}+R_{2}{ }^{0}$ $=11,9 \cdot 10^{-13} \mathrm{~cm}$ und $\lambda=40,71 \mathrm{MeV}$ ) folgende $T$.Ver-

2 W. E. Stein, Phys. Rev. 108, 94 [1957].

3 J. S. Fraser u. J. C. D. Milton, Proceedings of the Second

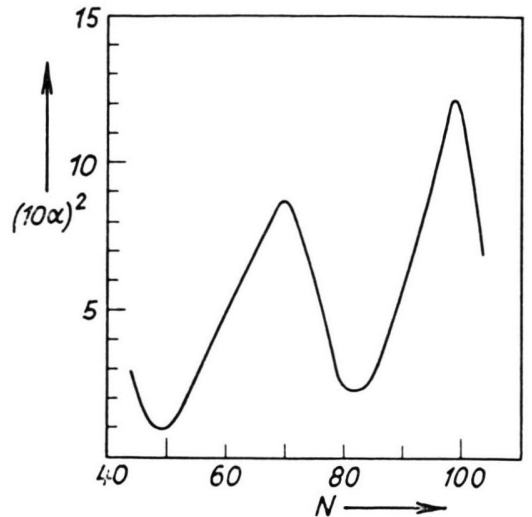

Abb. 3. Aus den $V_{\mathrm{K}}$-Werten der Abb. 2 nach Anm. ${ }^{1}$ berech nete Deformation eines Bruchstücks in Abhängigkeit von seiner Neutronenzahl $N$ bei der spontanen Spaltung von $\mathrm{Cf}^{252}$.

lauf ist in Abb. 1 mit eingezeichnet; die Úbereinstimmung mit den experimentellen Daten nach Fraser und Milton ${ }^{3}$ ist auch hier sehr gut.

Wir können somit feststellen, daß unsere Vorstellung der je nach der Schalenstruktur verschieden stark deformierten Bruchstücke sowohl zu einer Erklärung der Spaltungsasymmetrie (Anm. ${ }^{1}$ ) als auch des Verlaufs der kinetischen Energie $T$ der Fragmente führt. Damit erscheint der Versuch erfolgreich, die wesentlichen Charakteristika des Kernspaltungsprozesses als Folge des magischen Effektes in der Größe der Deformation der Bruchstücke zu verstehen.

Über die hier mitgeteilten Untersuchungen wird in Kürze an anderer Stelle ausführlicher berichtet werden.

United Nations International Conference on the Peaceful Uses of Atomic Energy, Geneva, P/199 [1958].

\section{Radiometrische Sedimentationsanalyse}

Von R. Lindner, J. O. Liljenzin und H. Reinhardt

Kernchemisches Institut der Technischen Hochschule Göteborg (Z. Naturforschg. 15 a, 1110-1112 [1960] ; eingeg. am 30. September 1960)

Bei Untersuchungen am hiesigen Institut (Diffusion von Spaltprodukten in Uranverbindungen ${ }^{1}$; Bestimmung der Größe von großen Radiokolloidteilchen ${ }^{2}$ ) ergab sich die Notwendigkeit, die Korngröße (oder die Dichte) kleiner Mengen $\alpha$-strahlender fester Stoffe zu bestimmen.

Der Gedanke lag nahe, die geringe Reichweite (max. $0,1 \mathrm{~mm}$ in Wasser) von $\alpha$-Strahlen zu einer „radiometrischen“ Sedimentationsmethode auszunutzen. Im

1 R. Lindner u. Hu. Matzke, Z. Naturforschg. 14 a, 1074 [1959]. vorliegenden Fall wird folgendes Prinzip verwendet: Bei Versuchsbeginn befindet sich das $\alpha$-strahlende Material aufgeschlämmt und homogen verteilt in einer Flüssigkeitssäule, um dann durch Sedimentation auf den Boden zu sinken und dort mit geeigneten Detektoren gemessen zu werden. Am einfachsten ist die direkte Befestigung des Sedimentationszylinders auf dem Endfenster eines Proportionalzählrohres, dessen Betriebsspannung so gewählt wird, daß nur $\alpha$-Strahlen registriert werden. Die Versuchsanordnung ist schematisch in Abb. 1 wiedergegeben.

Die Auswertung der Versuche basiert auf der Stokesschen Gleichung in der Form

$$
d=141 \sqrt{\frac{n h}{(\varrho-\varrho \mathrm{Fl}) t},},
$$

2 R. Lindner, J. O. Liljenzin u. H. Reinhardt, Z. Elektrochem. Ber. Bunsenges. physik. Chem., im Druck. 